\title{
Trajectory Controllability of Semilinear Differential Evolution Equations with Impulses and Delay
}

\author{
Maojun Bin, Yiliang Liu \\ College of Sciences, Guangxi University for Nationalities, Nanning 530006, Guangxi Province, P. R. China \\ Email: bmj1999@163.com, yiliangliu100@126.com
}

Received 2013

\begin{abstract}
This paper researches trajectory controllability of semilinear differential evolution equations with impulses and delay. The main techniques in our paper rely on the fixed point theorem and monotone operator theory. In the end of the paper, an example is given to explain our main result.
\end{abstract}

Keywords: Trajectory Controllability; Monotone Operator Theory; Mild Solution; Fixed Point Theorem; Lipschitz Continuity

\section{Introduction}

The impulsive differential systems originate from the real world problems to describe the dynamics of processes in which sudden, discontinuous jumps occurs. Impulsive differential equations have become more important in many mathematical models of real processes and phenomena studied in control, physics, chemistry, population dynamics, aeronautics and engineering. Because of their significance, many scholars have been researched the solvability of impulsive differential equations in recent years, especially in the area of impulsive differential equations with fixed moments, see the monographs of Bainov and Simeonov [3], Lakshmikantham et al. [14] and Samoilenko and Perestyuk [17] and the papers of $[1,2,4,9-13,22$. Another hand, differential equations with delay was initiated about existence and stability by Travis and Webb [19] and Webb [21]. Due to such equations are often more realistic to describe natural phenomena than those without delay, they have been investigated in different aspects by many authors $[2,15]$.

The concept of controllability (introduced by Kalman 1960) plays an important role in many areas of applied mathematics. In recent years, significant progress has been made in the controllability of linear and nonlinear deterministic systems $[8,16,18,20]$. But it does not give any idea about the control path along which trajectory moves.

In[7], D. N. Chalishajar, R. K. George, A. K. Nandakumaran, and F. D. Acharya studied trajectory controllability of the following fractional nonlinear integro-differential systems

$$
\left\{\begin{array}{l}
w^{\prime}(t)=A w(t)+B(t, u(t))+F(t, w(t), \\
\int_{0}^{t} G(t, s, w(s)) d s, t \in J=[0, T], \\
w(0)=w_{0},
\end{array}\right.
$$

where $H$ and $U$ are Hilbert spaces, the state $w(t) \in H$ and the control $u(t) \in U$, for each $t \in J$. The operator $A: \overline{D(A)} \subset H \mapsto H$ is a linear operator not necessarily bounded. The maps $B: J \times U \mapsto H, \quad G: \Delta \times H \mapsto H$ and $F: J \times H \times H \mapsto H$ are nonlinear operators, where $\Delta=\{(t, s) \in J \times J: 0 \leq s \leq t \leq T\}$.

Motivated by the above work, in this paper, we consider the following equation:

$\begin{cases}x^{\prime}(t)=A x(t)+B(t, u(t))+f\left(t, x_{t}\right) & t \in J=[0, T], t \neq t_{k}, \\ \Delta x\left(t_{k}\right)=I_{k}\left(x\left(t_{k}\right)\right), k=1,2, \cdots, m & x(t)=\varphi(t),-r \leq t \leq 0,\end{cases}$

where $J^{*}=[-r, T]$. Let $X$ be a real Banach space, the state $x(t) \in X$ and the control $u(t) \in L(J, U)$ is a Banach space of admissible control function with $U$ a Banach space, $A: D(A) \subset X \mapsto X$ is the infinitesimal generator of a $C_{0}$-semigroup $T(t), t>0$. The maps $B: J \times U \mapsto X$, and $f: J \times X \mapsto X$ are nonlinear operators. $D=\{\phi:[-r, 0] \mapsto X, \phi$ is continuous everywhere except for a finite number of points $s$ at which $\phi\left(s^{-}\right), \phi\left(s^{+}\right)$exist and $\left.\phi\left(s^{-}\right)=\phi(s)\right\}$. $\varphi \in D(0<r<\infty)$, for $\varphi \in D$ the norm of $\varphi$ is defined by $\|\varphi\|_{D}=\sup \{|\varphi(t)|:-r \leq t \leq 0\} . \quad 0=t_{0}<t_{1}<\cdots<t_{m}<t_{m+1}=T$, $I_{k}: X \mapsto X, \quad \Delta x\left(t_{k}\right)=x\left(t_{k}^{+}\right)-x\left(t_{k}^{-}\right), \quad x\left(t_{k}^{+}\right)$and $x\left(t_{k}^{-}\right)$ denote the right and the left limits of $x(t)$ at $t=t_{k}$, $k=1,2, \cdots, m$. For any continuous function $x$ defined on $J^{*} \mid\left\{t_{1}, t_{2}, \cdots, t_{m}\right\}$ and $t \in J$, we denote by $x_{t}$ the 
element of $D$ defined by $x_{t}(s)=x(t+s), \quad-r \leq s \leq 0$, here $x_{t}(\cdot)$ represents the history of the state from $t-r$, up to the present time $t$.

The rest of this paper is organized as follows: In section 2, we present some preliminaries to prove our main results. In section 3, by applying some standard fixed point principles, we prove the existence of the mild solutions for fractional nonlinear integro-differential equations. In section 4 , the trajectory controllability of the system (1.1) is proved by applying the tools of monotone operator theory and set-valued analysis. In section 5 , we give an example to illustrate our main results.

\section{Preliminaries}

In this section, we introduce definitions and preliminaries which are used throughout this paper, and then we give the mild solutions of systems (1.1).

Let $(X,\|\cdot\|)$ be a real Banach space. We denote by $C(J, X)$ the space of $X$-valued continuous function on $J$, with the norm $\|x\|=\sup \{|x|: T \in J\}$ and by $L^{1}(J, X)$ the space of $X$-valued Bochner integral functions on $J$ with the norm $\|f\|_{L^{1}}=\int_{0}^{T}|f(t)| d t$. Let

$P C(J, x)=\left\{x: J \mapsto X, x\right.$ is continuous at $t \neq t_{k}$ and the left continuous at $t=t_{k}$, the right limit $x\left(t_{k}^{+}\right)$exist, $k=1,2, \cdots, m\}$. It is easy to verify that $\operatorname{PC}(J, X)$ is a Banach space with the norm

$$
\|x\|_{P C}=\max \left\{\sup _{t \in J}\|x(t+0)\|, \sup _{t \in J}\|x(t-0)\|\right\}, B(x)
$$

denotes the Banach space of bounded linear operator from $X$ to $X$ with norm $\|F\|_{B(X)}=\sup \{|F(x)|:|x|=1\}$.

Definition 2.1. A function $x(\cdot) \in X$ is a solution (mild solution) of the system (1.1) if it satisfies

$$
\begin{aligned}
x(t)= & T(t) \varphi(0)+\int_{0}^{t} T(t-s)\left[B(s, u(s))+f\left(s, x_{s}\right)\right] d s \\
& +\sum_{0<t_{k}<t} T\left(t-t_{k}\right) I_{k}\left(x\left(t_{k}\right)\right) .
\end{aligned}
$$

Definition 2.2. The system (1.1) is said to be completely controllability on $J$ if for any $x_{0}, x_{1} \in R$, and fixed $T$, there exists a control $u \in L^{2}(J)$ such that the corresponding solution $x(\cdot)$ of (1.1) satisfied $x(T)=x_{1}$.

Let $\Gamma$ be the set of all functions $z(\cdot)$ defined on $J=[0, T]$ such that $z(0)=x_{0}, z(T)=x_{1}, t \in J$ and $z$ is differentiable almost everywhere.

Definition 2.3. The system (1.1) is said to be T-controllability if for any $z \in \Gamma$, there exists a control $u \in L^{2}(J)$ such that the corresponding solution $x(\cdot)$ of (1.1) satisfied $x(t)=z(t)$ a.e. $t \in J$.

Definition 2.4. The system (1.1) is totally controllable on $J$ if for all subinterval $\left[t_{i}, t_{f}\right] \subset[0, T], t_{i} \in J$ the system (2.1) is completely controllable.

Clearly, $\quad T$-controllability $\Rightarrow$ Total controllability $\Rightarrow$ Complete controllability.
Now, we give the following properties which would be used to our main result in the next.

Lemma 2.1.([5]) Let $X$ be a Banach space, and $P, Q: X \mapsto X$ two operators satisfying:

(i) $P$ is a contraction, and

(ii) $Q$ is completely continuous, then either

(a) the operator equation $x=P(x)+Q(x)$ has a solution, or

(b) the set $\eta=\left\{x \in X: \lambda P\left(\frac{x}{\lambda}\right)+\lambda Q(x)=x, \lambda \in(0,1)\right\}$ is unbounded.

Lemma 2.2. ([6])(Main Theorem on Monotone Operators) Let $X$ be a real, reflexive Banach space, and let $\Phi: X \mapsto X^{*}$ be a monotone, hemicontinuous, bounded, and coercive operator, and $b \in X^{*}$. Then there exists a solution of the equation $\Phi u=b$.

\section{Existence of Mild Solutions}

In this section we prove the existence and uniqueness of the mild solution of problem (1.1). Before stating and proving the result, we assume the following conditions hold:

$H(1)$ : There exist a constant $M \geq 0$ such that

$$
M=\sup \left\{\|T\|_{B(X)}<\infty, t \in J\right\} .
$$

$H(2)$ : $B$ satisfies Caratheadory condition, i.e.,

$$
B(t, \cdot): U \mapsto X
$$

is continuous for $t \in J$ and $B(\cdot, y): J \mapsto X$ is measurable for $y \in U$.

$H(3)$ : $f$ satisfies Caratheadory conditions like $B$, i.e., $f(t, \cdot): X \mapsto X \quad$ is continuous for $t \in J$ and $f(\cdot, x): J \mapsto X$ is measurable for $x \in X$.

$H(4)$ : There exist two functions $a_{0}(t), b_{0}(t) \in L^{1}(J, X)$ and two constants $a_{1}, b_{1}>0$, such that $B$ and $f$ satisfy following growth conditions:

$$
\begin{gathered}
\|B(t, u)\|_{X} \leq b_{0}(t)+b_{1}\|u\|_{U}, \forall u \in U, t \in J, \\
\|f(t, x)\|_{X} \leq a_{0}(t)+a_{1}\|x\|_{X}, \forall t \in J, x \in X .
\end{gathered}
$$

$H(5): f(t, x)$ is Lipschitz continuous with respect to $X$, i.e. there exist constants $\alpha \geq 0$ such that

$$
\left\|f\left(t_{1}, x_{1}\right)-f\left(t_{2}, x_{2}\right)\right\| \leq \alpha_{1}\left\|x_{1}-x_{2}\right\|,
$$

for all $x_{1}, x_{2} \in X, t \in J$.

$H(6)$ : there exist constants $d_{k}>0, k=1,2, \cdots, m$, with $M \sum_{k=1}^{m} d_{k}<1$ such that

$$
\left|I_{k}(x)-I_{k}(y)\right| \leq d_{k}|x-y|, \forall x, y \in X .
$$

Now, let us begin prove the existence and uniqueness of the mild solution of (1.1).

Theorem 3.1. If the conditions $H(1)-H(6)$ hold, then the problem (1.1) has at least one mild solution on $X$. 
Proof. Transform the problem (1.1) into a fixed problem. Consider the two operators $P, Q: P C\left(J^{*}, X\right) \mapsto P C\left(J^{*}, X\right)$ defined by

$$
P x(t)=\left\{\begin{array}{c}
0, \quad t \in[-r, 0], \\
\sum_{0<t_{k}<t} T\left(t-t_{k}\right) I_{k}\left(x\left(t_{k}\right)\right), t \in J,
\end{array}\right.
$$

and

$$
Q x(t)=\left\{\begin{array}{l}
\varphi(t), \quad t \in[-r, 0] \\
T(t) \varphi(0)+\int_{0}^{t} T(t-s)\left[B(s, u(s))+f\left(s, x_{s}\right)\right] d s \\
t \in J .
\end{array}\right.
$$

Then the problem of finding the solution of problem (1.1) is reduced to finding the solution of the operator equation $P x(t)+Q x(t)=x(t), t \in J^{*}$. We shall show that the operators $P$ and $Q$ satisfy all the conditions of Lemma 2.1. For better readability, we break the proof into a sequence of steps.

Step 1. $Q$ is continuous.

Let $\left\{x_{n}\right\}$ be a sequence such that $x_{n} \rightarrow x$ in $P C\left(J^{*}, x\right)$, then for $t \in J$, we have

$$
\begin{aligned}
\left|Q x_{n}(t)-Q x(t)\right| & \leq\left|\int_{0}^{t} T(t-s)\left[f\left(s, x_{n_{s}}\right)-f\left(s, x_{s}\right)\right] d s\right| \\
& \leq M \int_{0}^{t}\left|f\left(s, x_{n_{s}}\right)-f\left(s, x_{s}\right)\right| d s .
\end{aligned}
$$

Since $f(s, \cdot)$ is continuous for a.e. $s \in J$, by the Lebesgue dominated convergence theorem, we have

$$
\left|Q x_{n}(t)-Q x(t)\right| \rightarrow 0, \text { as } n \rightarrow \infty .
$$

Thus $Q$ is continuous.

Step 2. $Q$ maps bounded sets into bounded sets in $P C\left(J^{*}, X\right)$.

It is enough to show that for any $\gamma>0$ there exists a positive constant $l$ such that for each $x \in B_{\gamma}=\left\{x \in P C\left(J^{*}, X\right):\|x\| \leq l\right\}$, we have $\|Q x\| \leq l$. So we choose $x \in B_{\gamma}$, then for each $t \in J$, we have

$$
\begin{aligned}
|Q x(t)| & \leq\left|T(t) \varphi(0)+\int_{0}^{t} T(t-s)\left[B(s, u(s))+f\left(s, x_{s}\right)\right] d s\right| \\
& \leq M|\varphi(0)|+M \int_{0}^{t}\left[b_{0}(s)+b_{1}|u(s)|+a_{0}(s)\right. \\
& \left.+a_{1}\left|x_{s}\right|\right] d s \\
& \leq M|\varphi(0)|+M \int_{0}^{t}\left[b_{0}(s)+a_{0}(s)\right] d s+M\left[a_{1}\|x\|_{L^{1}}\right. \\
& \left.+b_{1}\|u\|_{L^{1}}\right] .
\end{aligned}
$$

As $a_{0}(t), b_{0}(t) \in L^{1}(J, X)$, then we have

$$
\begin{aligned}
& \|Q x\| \leq M\|\varphi\|+M \int_{0}^{t}\left[b_{0}(s)+a_{0}(s)\right] d s \\
& +a_{1}\|x\|_{L^{1}}+b_{1}\|u\|_{L^{1}}=l .
\end{aligned}
$$

Step 3. $Q$ maps bounded sets into equicontinuous sets of $P C\left(J^{*}, X\right)$

We consider $B_{\gamma}$ as in step 2 and let $\tau_{1}, \tau_{2} \in J /\left\{t_{1}, t_{2}, \cdots, t_{m}\right\}$, $\tau_{1}<\tau_{2}$. Thus if $\varepsilon>0$ and $\varepsilon<\tau_{1}<\tau_{2}$, we have

$\left|Q x\left(\tau_{2}\right)-Q x\left(\tau_{1}\right)\right|$

$\leq\left|T\left(\tau_{2}\right) \varphi(0)-T\left(\tau_{1}\right) \varphi(0)\right|$

$+\left|\int_{0}^{\tau_{2}} T\left(\tau_{2}-s\right) B(s, u(s)) d s-\int_{0}^{\tau_{1}} T\left(\tau_{1}-s\right) B(s, u(s)) d s\right|$

$+\left|\int_{0}^{\tau_{2}} T\left(\tau_{2}-s\right) f\left(s, x_{s}\right) d s-\int_{0}^{\tau_{1}} T\left(\tau_{1}-s\right) f\left(s, x_{s}\right) d s\right|$

$\leq\left|T\left(\tau_{2}\right) \varphi(0)-T\left(\tau_{1}\right) \varphi(0)\right|$

$+\int_{0}^{\tau_{1}-\varepsilon}\left|T\left(\tau_{2}-s\right)-T\left(\tau_{1}-s\right)\right|\left[b_{0}(s)+b_{1}|u(s)|\right] d s$

$+\int_{\tau_{1}-\varepsilon}^{\tau_{1}}\left|T\left(\tau_{2}-s\right)-T\left(\tau_{1}-s\right)\right|\left[b_{0}(s)+b_{1}|u(s)|\right] d s$

$+\int_{\tau_{1}}^{\tau_{2}}\left|T\left(\tau_{2}-s\right)\right|\left[b_{0}(s)+b_{1}|u(s)|\right] d s$

$+\int_{0}^{\tau_{1}-\varepsilon}\left|T\left(\tau_{2}-s\right)-T\left(\tau_{1}-s\right)\right|\left[a_{0}(s)+a_{1}\left|x_{s}\right|\right] d s$

$+\int_{\tau_{1}-\varepsilon}^{\tau_{1}}\left|T\left(\tau_{2}-s\right)-T\left(\tau_{1}-s\right)\right|\left[a_{0}(s)+a_{1}\left|x_{s}\right|\right] d s$

$+\int_{\tau_{1}}^{\tau_{2}}\left|T\left(\tau_{2}-s\right)\right|\left[a_{0}(s)+a_{1}\left|x_{s}\right|\right] d s$

$=Q_{1}+Q_{2}+Q_{3}+Q_{4}+Q_{5}+Q_{6}+Q_{7}$.

We easily get,

$$
\begin{aligned}
Q_{1}= & \left|T\left(\tau_{2}\right) \varphi(0)-T\left(\tau_{1}\right) \varphi(0)\right| \rightarrow 0, \text { as } \tau_{1} \rightarrow \tau_{2}, \\
Q_{2}= & \int_{0}^{\tau_{1}-\varepsilon}\left|T\left(\tau_{2}-s\right)-T\left(\tau_{1}-s\right)\right|\left[b_{0}(s)+b_{1}|u(s)|\right] d s \\
\leq & \sup _{s \in\left[0, \tau_{1}-\varepsilon\right]}\left\|T\left(\tau_{2}-s\right)-T\left(\tau_{1}-s\right)\right\| \\
& \times\left[\int_{0}^{\tau_{1}-\varepsilon} b_{0}(s) d s+b_{1}\|u\|_{L^{1}}\right] \rightarrow 0, \\
\text { as } & \tau_{1} \rightarrow \tau_{2}, \varepsilon \rightarrow 0, \\
Q_{3}= & \int_{\tau_{1}-\varepsilon}^{\tau_{1}-\varepsilon}\left|T\left(\tau_{2}-s\right)-T\left(\tau_{1}-s\right)\right|\left[b_{0}(s)+b_{1}|u(s)|\right] d s \\
\leq & \sup _{s \in\left[\tau_{1}-\varepsilon, \tau_{1}\right]}\left\|T\left(\tau_{2}-s\right)-T\left(\tau_{1}-s\right)\right\| \\
& \times\left[\int_{\tau_{1}-\varepsilon}^{\tau_{1}} b_{0}(s) d s+b_{1}\|u\|_{L^{1}}\right] \rightarrow 0, \\
\text { as } & \tau_{1} \rightarrow \tau_{2}, \varepsilon \rightarrow 0, \\
Q_{4}= & \int_{\tau_{1}}^{\tau_{2}}\left|T\left(\tau_{2}-s\right)\right|\left[b_{0}(s)+b_{1}|u(s)|\right] d s \\
\leq & M\left(\int_{\tau_{1}}^{\tau_{2}}\left[b_{0}(s)+b_{1}|u(s)|\right] d s \rightarrow 0,\right. \\
\text { as } & \tau_{1} \rightarrow \tau_{2}, \\
Q_{5}= & \int_{0}^{\tau_{1}-\varepsilon}\left|T\left(\tau_{2}-s\right)-T\left(\tau_{1}-s\right)\right|\left[a_{0}(s)+a_{1}\left|x_{s}\right|\right] d s \\
\leq & \sup _{s \in\left[0, \tau_{1}-\varepsilon\right]}\left\|T\left(\tau_{2}-s\right)-T\left(\tau_{1}-s\right)\right\| \\
& \times\left[\int_{0}^{\tau_{1}-\varepsilon} a_{0}(s) d s+a_{1}\|x\|_{L^{1}}\right] \rightarrow 0, \\
\text { as } & \delta \rightarrow 0, \\
Q_{6}= & \int_{\tau_{1}-\varepsilon}^{\tau_{1}}\left|T\left(\tau_{2}-s\right)-T\left(\tau_{1}-s\right)\right|\left[a_{0}(s)+a_{1}\left|x_{s}\right|\right] d s \\
\leq & \sup _{\left.\operatorname{sef} \tau_{1}-\varepsilon, \tau_{1}\right]}\left\|T\left(\tau_{2}-s\right)-T\left(\tau_{1}-s\right)\right\| \\
& \times\left[\int_{\tau_{1}-\varepsilon}^{\tau_{1}} a_{0}(s) d s+a_{1}\|x\|_{L^{1}}\right] \rightarrow 0, \\
\text { as } & \tau_{1} \rightarrow \tau_{2}, \varepsilon \rightarrow 0,
\end{aligned}
$$




$$
\begin{aligned}
Q_{7} & =\int_{\tau_{1}}^{\tau_{2}}\left|T\left(\tau_{2}-s\right)\right|\left[a_{0}(s)+a_{1}\left|x_{s}\right|\right] d s \\
& \leq M\left(\int_{\tau_{1}}^{\tau_{2}}\left[a_{0}(s)+a_{1}\left|x_{s}\right|\right] d s \rightarrow 0, \text { as } \delta \rightarrow 0,\right.
\end{aligned}
$$

Then, we get $\left\|Q x\left(\tau_{2}\right)-Q x\left(\tau_{1}\right)\right\| \rightarrow 0 \quad$ as $\quad \tau_{1} \rightarrow \tau_{2}$, $\varepsilon \rightarrow 0$, since $T(t)$ is a strongly continuous operator and the compactness of $T(t)$ for $t>0$ implies the continuity in the uniform operator topology. This proves the equicontinuity for the case where $t \neq t_{k}, k=1,2, \cdots, m$. It remains to examine the equicontinuity at $t=t_{k}$.

First, we prove equicontinuity at $t=t_{i}^{-}$. Fixed $\delta_{1}>0$ such that $\left\{t_{k}: k \neq i\right\} \bigcap\left[t_{i}-\delta_{1}, t_{i}+\delta_{1}\right]=\varnothing$. For $0<h<\delta_{1}$ we have

$$
\begin{aligned}
& \left|Q x\left(t_{i}\right)-Q x\left(t_{i}-h\right)\right| \\
\leq & \left|T\left(t_{i}\right) \varphi(0)-T\left(t_{i}-h\right) \varphi(0)\right| \\
& +\int_{0}^{t_{i}-h}\left|T\left(t_{i}-s\right)-T\left(t_{i}-h-s\right)\right|\left[b_{0}(s)+b_{1}|u(s)|\right] d s \\
& +M \int_{t_{i}-h}^{t_{i}}\left[b_{0}(s)+b_{1}|u(s)|\right] d s \\
& +\int_{0}^{t_{i}-h}\left|T\left(t_{i}-s\right)-T\left(t_{i}-h-s\right)\right|\left[a_{0}(s)+a_{1}\left|x_{s}\right|\right] d s \\
& +M \int_{t_{i}-h}^{t_{i}}\left[a_{0}(s)+a_{1}\left|x_{s}\right|\right] d s,
\end{aligned}
$$

which tends to zero as $h \rightarrow 0$.

Define

$$
\widetilde{Q_{0}} x(t)=Q x(t), t \in\left[0, t_{1}\right],
$$

and

$$
\widetilde{Q_{i}} x(t)=\left\{\begin{array}{c}
Q x(t), t \in\left(t_{i} \cdot t_{i+1}\right], \\
Q x\left(t_{i}^{+}\right), t=t_{i} .
\end{array}\right.
$$

Next, we prove equicontinuity at $t=t_{i}^{+}$. Fixed $\delta_{2}>0$ such that $\left\{t_{k}: k \neq i\right\} \bigcap\left[t_{i}-\delta_{2}, t_{i}+\delta_{2}\right]=\varnothing$. For $0<h<\delta_{2}$ we have

$$
\begin{aligned}
& \quad\left|Q x\left(t_{i}+h\right)-Q x\left(t_{i}\right)\right| \\
& \leq\left|T\left(t_{i}+h\right) \varphi(0)-T\left(t_{i}\right) \varphi(0)\right| \\
& +\int_{0}^{t_{i}}\left|T\left(t_{i}+h-s\right)-T\left(t_{i}-s\right)\right|\left[b_{0}(s)+b_{1}|u(s)|\right] d s \\
& +M \int_{t_{i}}^{t_{i}+h}\left[b_{0}(s)+b_{1}|u(s)|\right] d s \\
& +\int_{0}^{t_{i}}\left|T\left(t_{i}+h-s\right)-T\left(t_{i}-s\right)\right|\left[a_{0}(s)+a_{1}\left|x_{s}\right|\right] d s \\
& +M \int_{t_{i}}^{t_{i}+h}\left[a_{0}(s)+a_{1}\left|x_{s}\right|\right] d s,
\end{aligned}
$$

which tends to zero as $h \rightarrow 0$. The equicontnuity for the cases $\tau_{1}<\tau_{2} \leq 0$ and $\tau_{1} \leq 0<\tau_{2}$ follows from the uniform continuity of $\varphi$ on the interval $[-r, 0]$.

As consequence of Steps 1 to 3 together with Arzela-Ascoli theorem it suffices to show that $B$ maps $B$ into a precompact set in $X$.

Let $0<t<T$ be fixed and let $0<\varepsilon<t$ be a real number. For $x \in B_{\gamma}$ we define

$$
\begin{aligned}
Q_{\varepsilon} x(t)= & T(t) \varphi(0)+T(\varepsilon) \int_{0}^{t-\varepsilon} T(t-s-\varepsilon)[B(s, u(s)) \\
& \left.+f\left(s, x_{s}\right)\right] d s .
\end{aligned}
$$

Since $T(t)$ is a compact operator, the set $X_{\varepsilon}(t)=$ $\left\{Q_{\varepsilon} x(t): x \in B_{\gamma}\right\}$ is precompact in $X$ for every $\varepsilon$, $0<\varepsilon<t$. Moreover, for every $x \in B_{\gamma}$, we have

$$
\begin{aligned}
\left|Q x(t)-Q_{\varepsilon} x(t)\right| \leq & \int_{t-\varepsilon}^{t}\|T(t-s)\|\left[a_{0}(s)+a_{1}\left|x_{s}\right|\right. \\
& \left.+b_{0}(s)+b_{1}|u(s)|\right] d s .
\end{aligned}
$$

Therefore, there are precompact sets arbitrarily close to the set $X_{\varepsilon}(t)=\left\{Q_{\varepsilon} x(t): x \in B_{\gamma}\right\}$. Hence the set $X_{\varepsilon}(t)=\left\{Q_{\varepsilon} x(t): x \in B_{\gamma}\right\}$ is precompact in $X$. Hence the operator $Q: P C\left(J^{*}, X\right) \mapsto P C\left(J^{*}, X\right)$ is completely continuous.

Step 4. $P$ is a contraction.

Let $x, y \in P C\left(J^{*}, X\right)$, then for $t \in J$, we have

$$
\begin{aligned}
& |P x(t)-P y(t)| \\
= & \left|\sum_{0<t_{k}<t} T\left(t-t_{k}\right) I_{k}\left(x\left(t_{k}\right)\right)-\sum_{0<t_{k}<t} T\left(t-t_{k}\right) I_{k}\left(y\left(t_{k}\right)\right)\right| \\
\leq & M \sum_{0<t_{k}<t}\left|I_{k}\left(x\left(t_{k}\right)\right)-I_{k}\left(y\left(t_{k}\right)\right)\right| \\
\leq & M \sum_{0<t_{k}<t} d_{k}\left|x\left(t_{k}\right)-y\left(t_{k}\right)\right| \leq M \sum_{0<t_{k}<t} d_{k}\|x-y\|,
\end{aligned}
$$

i.e., $\quad\|P x(t)-P y(t)\| \leq M \sum_{0<t_{k}<t} d_{k}\|x-y\|$,

since $M \sum_{0<t_{k}<t} d_{k}<1$, then $P$ is a contraction.

Step 5. A priori bounds.

It remains to show that the set

$\eta=\left\{x \in P C\left(J^{*}, X\right): x=\lambda Q x+\lambda P \frac{x}{\lambda}\right.$ for some $\left.0<\lambda<1\right\}$, is bounded.

Let $x \in \eta$, then $x=\lambda Q x+\lambda P \frac{x}{\lambda}$ for some $0<\lambda<1$. Thus for each $t \in J$,

$$
\begin{aligned}
x(t) & =\lambda T(t) \varphi(0)+\lambda \int_{0}^{t} T(t-s)\left[B(s, u(s))+f\left(s, x_{s}\right)\right] d s \\
& +\lambda \sum_{0<t_{k}<t} T\left(t-t_{k}\right) I_{k}\left(\frac{x}{\lambda}\left(t_{k}\right)\right) .
\end{aligned}
$$

Implying $H(4)$ and $H(6)$, for each $t \in J$, we have

$$
\begin{aligned}
|x(t)| \leq & \lambda M|\varphi(0)|+\lambda M \int_{0}^{t}\left[b_{0}(s)+a_{0}(s)\right] d s \\
+ & \lambda a_{1} M\|x\|_{L^{1}}+\lambda b_{1} M\|u\|_{L^{1}}+\lambda M \sum_{k=1}^{m}\left|I_{k}\left(\frac{x}{\lambda}\left(t_{k}\right)\right)\right| \\
\leq & \lambda M\|\varphi\|+\lambda M \int_{0}^{t}\left[b_{0}(s)+a_{0}(s)\right] d s \\
+ & \lambda a_{1} M\|x\|_{L^{1}}+\lambda b_{1} M\|u\|_{L^{1}} \\
+ & \lambda M \sum_{k=1}^{m}\left|I_{k}\left(\frac{x}{\lambda}\left(t_{k}\right)\right)-I_{k}(0)\right|+\lambda M \sum_{k=1}^{m}\left|I_{k}(0)\right| \\
\leq & \lambda M\left(\|\varphi\|+\sum_{k=1}^{m}\left|I_{k}(0)\right|\right) \\
& +\lambda M \int_{0}^{t}\left[b_{0}(s)+a_{0}(s)\right] d s+\lambda a_{1} M\|x\|_{L^{1}} \\
& +\lambda b_{1} M\|u\|_{L^{1}}+\lambda M \sum_{k=1}^{m} d_{k}\left|\frac{x}{\lambda}\left(t_{k}\right)\right| \\
\leq & M\left(\|\varphi\|+\sum_{k=1}^{m}\left|I_{k}(0)\right|\right)+M \int_{0}^{t}\left[b_{0}(s)+a_{0}(s)\right] d s \\
& +M\left[a_{1}\|x\|_{L^{1}}+b_{1}\|u\|_{L^{1}}+M \sum_{k=1}^{m} d_{k}\left|x\left(t_{k}\right)\right|\right]
\end{aligned}
$$


Now we consider the function $\mu$ defined by

$$
\mu(t)=\sup \{|x(s)|:-r \leq s \leq t\}, 0 \leq t \leq T .
$$

Then $\left\|x_{s}\right\| \leq \mu(t)$ for all $t \in J$ and there is a point $t^{*} \in[-r, t]$ such that $\mu(t)=\left|x\left(t^{*}\right)\right|$. If $t^{*} \in J$, by the previous inequality we have for $t \in J$ ( note $t^{*} \leq t$

$$
\begin{aligned}
\mu(t) \leq & M\left(\|\varphi\|+\sum_{k=1}^{m}\left|I_{k}(0)\right|\right)+M \int_{0}^{t}\left[b_{0}(s)+a_{0}(s)\right] d s \\
& +M\left[a_{1}\|x\|_{L^{1}}+b_{1}\|u\|_{L^{1}}+M \sum_{k=1}^{m} d_{k} \mu(t)\right]
\end{aligned}
$$

i.e.,

$$
\begin{aligned}
\left(1+M \sum_{k=1}^{m} d_{k}\right) \mu(t) \leq & M\left(\|\varphi\|+\sum_{k=1}^{m}\left|I_{k}(0)\right|\right) \\
& +M \int_{0}^{t}\left[b_{0}(s)+a_{0}(s)\right] d s \\
& +M\left[a_{1}\|x\|_{L^{1}}+b_{1}\|u\|_{L^{1}}\right] .
\end{aligned}
$$

Hence there exists a constant $K$ such that,

$$
\begin{aligned}
\mu(t) \leq & \frac{1}{1+M \sum_{k=1}^{m} d_{k}}\left\{M\left(\|\varphi\|+\sum_{k=1}^{m}\left|I_{k}(0)\right|\right)\right. \\
& +M \int_{0}^{T}\left[b_{0}(s)+a_{0}(s)\right] d s \\
& \left.+M\left[a_{1}\|x\|_{L^{1}}+b_{1}\|u\|_{L^{1}}\right]\right\}=K .
\end{aligned}
$$

By the definition of $\mu$, we have

$$
\|x\|=\sup _{t \in J^{*}}|x(t)| \leq \mu(T) \leq K, \forall x \in \eta
$$

This shows that the set $\eta$ is bounded. As a consequence of Theorem 3.1 we deduce that $P+Q$ has a fixed point which is a mild solution of problem (1.1).

The proof is completed.

\section{T-Controllability Results}

In this section, we are concerned with the trajectory controllability of semilinear differential evolution equations with impulses and delay.

We make the following additional assumption on $B$ :

$H(7)$ : $B$ satisfies monotonicity and coercivity conditions, i.e.,

$$
<B(t, u)-B(t, v), u-v>\geq 0, \forall u, v \in U, t \in J,
$$

and

$$
\lim _{\|u\| \rightarrow \infty} \frac{<B(t, u), u>}{\|u\|}=\infty .
$$

Now let begin proving the $T$-controllability results for the problem (1.1).

Theorem 4.1. Under the conditions $H(1)-H(7)$, the problem (1.1) is $T$-controllable.

Proof. Let $z \in T$ be the prescribed trajectory with $z(0)=\varphi(0)$, we want to find a control $u$ satisfying

$$
\begin{aligned}
z(t)= & T(t) \varphi(0)+\int_{0}^{t} T(t-s)\left[B(s, u(s))+f\left(s, z_{s}\right)\right] d s \\
& +\sum_{0<t_{k}<t} T\left(t-t_{k}\right) I_{k}\left(z\left(t_{k}\right)\right) .
\end{aligned}
$$

The equation (4.1) can be written as

$$
\begin{aligned}
& z(t)-T(t) \varphi(0)-\int_{0}^{t} T(t-s) f\left(s, z_{s}\right) d s \\
& -\sum_{0<t_{k}<t} T\left(t-t_{k}\right) I_{k}\left(z\left(t_{k}\right)\right) \\
= & \int_{0}^{t} T(t-s) B(s, u(s)) d s .
\end{aligned}
$$

Differentiating with respect to $t$, we get

$$
\begin{aligned}
& z^{\prime}(t)-A T(t) \varphi(0)-\int_{0}^{t} A T(t-s) f\left(s, z_{s}\right) d s-f\left(t, z_{t}\right) \\
& -\sum_{0<t_{k}<t} A T\left(t-t_{k}\right) I_{k}\left(z\left(t_{k}\right)\right) \\
= & \int_{0}^{t} A T(t-s) B(s, u(s)) d s+B(t, u(t)) .
\end{aligned}
$$

Equation (4.3) can be written as

$$
y(t)=\int_{0}^{t} g(t, s) y(s) d s+y_{0}(t),
$$

where $y(t)=B(t, u(t)), g(t, s)=-A T(t-s)$ and

$$
\begin{aligned}
y_{0}(t)= & z^{\prime}(t)-A T(t) \varphi(0) \\
& -\int_{0}^{t} A T(t-s) f\left(s, z_{s}\right) d s-f\left(t, z_{t}\right) \\
& -\sum_{0<t_{k}<t} A T\left(t-t_{k}\right) I_{k}\left(z\left(t_{k}\right)\right) .
\end{aligned}
$$

Define an operator $\digamma: L^{2}(J, X) \mapsto L^{2}(J, X)$ by

$$
\digamma y(t)=\int_{0}^{t} g(t, s) y(s) d s .
$$

We easily know that $y_{0}(t)$ is continuous in $J, g(t, s)$ is continuous in $J \times J$, then for any $y_{1}, y_{2} \in L^{2}(J, X)$, we have

$$
\begin{aligned}
& \left\|\digamma y_{1}-\digamma y_{2}\right\| \\
= & \left\|\int_{0}^{t} g(t, s) y_{1}(s) d s-\int_{0}^{t} g(t, s) y_{2}(s) d s\right\| \\
\leq & \max _{0 \leq t \leq T} \int_{0}^{T}|g(t, s)| \cdot\left|y_{1}(s)-y_{2}(s)\right| d s \\
\leq & L\left\|y_{1}-y_{2}\right\| .
\end{aligned}
$$

$\digamma^{n}$ is a contraction for sufficiently large $n$. Hence by generalized Banach contraction principle, there exists a unique solution $y$ for (4.4) for given $y_{0} \in L^{2}(J, X)$. Therefore, $T$-controllability follows if we can extract $u(t)$ from the relation

$$
y(t)=B(t, u(t)) .
$$

To see this, define an operator $N: L^{2}(J, X) \mapsto L^{2}(J, X)$ by

$$
N u(t)=B(t, u(t)) \text {. }
$$

By the conditions $H(1)$ and $H(4), \mathrm{N}$ is well-defined, continuous and bounded operator. Also, $B(t, u(t))$ is monotone and coercive, then we can easily get $N$ is monotone and coercive. A hemi-continuous monotone mapping is of type $(M)$. The nonlinear map $N$ is onto. By Lemma 2.2, there exists a control $u$ satisfying (4.6). The measurability of $u(t)$ follows as $u$ is in $L^{2}(J, X)$. This proves $T$ - controllability of the problem (1.1). 
The proof is completed.

\section{Application}

Example. We consider the following semilinear impulsive equation:

$$
\left\{\begin{array}{l}
\frac{\partial}{\partial t} w(t, x)=\Delta w+u(t, x)+\frac{1}{3}\left[\cos ^{2} x(t)+\cos w(t)\right] \\
\quad t \in J=[0, T], t \neq t_{k} \\
w(0, x)=\varphi(0, x), \operatorname{in} \Omega, k=1,2, \cdots, m \\
w(t, x)=\varphi(t, x), \operatorname{in} \partial \Omega \times J \\
w\left(t_{k}^{+}, x\right)-w\left(t_{k}^{-}, x\right)=I_{k}\left(w\left(t_{k}, x\right)\right)
\end{array}\right.
$$

where $\Omega$ is abounded domain in $R^{n}(n \geq 1)$ with smooth boundary $\partial \Omega, \varphi(0, x), \varphi(t, x) \in L^{2}(\Omega)$.

We take $X=L^{2}(\Omega)$ and define the operator

$$
A: D(A) \subset X \mapsto X \text { by }
$$

$$
D(A)=H^{2}(\Omega) \bigcap H_{0}^{1}(\Omega), A w=-A(x, D) w .
$$

It is easily turned out that the operator $A$ generates equicontinuous $C_{0}$-semigroup on $X$. re the control term $B(t, u(t))=u(t)$ is linear,

$$
f\left(t, x_{t}\right)=\frac{1}{3}\left[\cos ^{2} x(t)+\cos w(t)\right]
$$

is Lipchitz conditions. It satisfies the conditions of Theorem 4.1, then the problem (5.1) is T-controllable.

\section{Acknowledgements}

This work was financially supported by NNSF of China Grant No.11271087, No.61263006, Guangxi Scientific Experimental (China- ASEAN Research) Centre No. 20 120116, open fund of Guangxi Key laboratory of hybrid computation and IC design analysis No.2012HC IC07, and the Innovation Project of Guangxi Graduate Education No. YCSZ2012062.

\section{REFERENCES}

[1] A. Anguraj and M. Mallika Arjunan, "Existence and Uniqueness of Mild and Classical Solutions of Impulsive Evolution Equations," Electronic Journal of Differential Equations, Vol. 111, 2005, pp. 1-8.

[2] A. Anguraj and M. Mallika Arjunan, "Existence Results for an Impulsive Neutral Integro-differential Equations in Banach Spaces," Nonlinear Study, Vol. 16, No. 1, 2009, pp. 33-48.

[3] D. D. Bainov and P. S. Simeonov, "Impulsive Differential Equations: Periodic Solutions and Applications," Longman Scientific and Technical Group, England, 1993.

[4] M. Benchohra J. Henderson and S. K. Ntouyas, "Existence Results for Impulsive Multivalued Semilinear Neutral Functional Inclusions in Banach Spaces," Journal of
Mathematical Analysis and Applications, Vol. 263, No. 2, 2001, pp. 763-780.

doi:10.1006/jmaa.2001.7663

[5] T. A. Burton and Colleen Kirk, "A Fixed Point Theorem of Krasnoselskiii-Schaefer Type," Mathematische Nachrichten, Vol. 189, No. 1, 1998, pp. 23-31. doi:10.1002/mana.19981890103

[6] S. Carl and S. Heikkila, "Fixed Point Theory in Ordered Sets and Applications," Springer, New York, Dordrecht, Heidelberg, London, 2010.

[7] D. N. Chalishajar, R. K. George, A. K. Nandakumaran and F. S. Acharya, "Trajectory Controllability of Nonlinear Integro-differential System," Journal of Frankin Insitute., Vol. 347, No. 7, 2010, pp. 1065-1075. doi:10.1016/j.jfranklin.2010.03.014

[8] L. Chen and G. Li, "Approximate Controllability of Impulsive Differential Equations with Nonlocal Conditions," International Journal of Nonlinear Science, Vol. 10, 2010, pp. 438-446.

[9] Z. Fan, "Impulsive Problems for Semilinear Differential Equations with Nonlocal Conditions," Nonlinear Analysis, Vol. 72, No. 2, 2010, pp. 1104-1109. doi:10.1016/j.na.2009.07.049

[10] Z. Fan and G. Li, "Existence Results for Semilinear Differential Equations with Nonlocal and Impulsive Conditions," Journal of Functional Analysis, Vol. 258, No. 5, 2010, pp. 1709-1727. doi:10.1016/j.jfa.2009.10.023

[11] E. Hernandez, M. Pierri and G. Goncalves, "Existence Results for an Impulsive Abstract Partial Differential Equation with State-dependent Delay," Computer Mathematic Application, Vol. 52, No. 3-4, 2006, pp. 411-420. doi:10.1016/j.camwa.2006.03.022

[12] E. Hernandez, M. M. Rabello and H. Henriaquez, "Existence of Solutions for Impulsive Partial Neutral Functional Differential Equations," Journal of Mathematic Analysis and Application, Vol. 331, No. 2, 2007, pp. $1135-1158$. doi:10.1016/j.jmaa.2006.09.043

[13] S. Ji and S. Wen, "Nonlocal Cauchy Problem for Impulsive Differential Equations in Banach Spaces," International Journal of Nonlinear Science, Vol. 10, 2010, pp. 88-95.

[14] V. Lakshmikantham, D. D. Bainov and P. S. Simeonov, "Theory of Impulsive Differential Equations," World Scientific, Singapore, 1989.

[15] M. Li, M. Wang and F. Zhang, "Controllability of Impulsive Functional Differential Systems in Banach Spaces," Chaos, Solitons and Fractals, Vol. 29, No. 1, 2006, pp. 175-181. doi:10.1016/j.chaos.2005.08.041

[16] Z. H. Liu and X. W. Li, "On the Controllability of Impulsive Fractional Evolution Inclusions in Banach Spaces," Journal of Optimizition Theory and Application, Vol. 156, No. 1, 2013, pp. 167-182. doi:10.1007/s10957-012-0236-x

[17] A. M. Samoilenko and N. A. Perestyuk, "Impulsive Differential Equations," World Scientific, Singapore, 1995.

[18] V. Obukhovski and P. Zecca, "Controllability for Systems Governed by Semilinear Differential Inclusions in a 
Banach Space with a Noncompact Semigroup," Nonlinear Analysis, Vol. 70, No. 9, 2009, pp. 3424-3436. doi:10.1016/j.na.2008.05.009

[19] C. Travis, G. Webb, "Existence and Stability for Partial Functional Differential Equations," Transactions of American Mathematical Society, Vol. 200, 1974, pp. 395-418. doi:10.1090/S0002-9947-1974-0382808-3

[20] F. Wang, Z. H. Liu and J. Li, "Complete Controllability of Fractional Neutral Differential Systems in Abstract Space, Abstract and Applied Analysis," Article ID
529025,2013, pp. 1-11.

[21] G. Webb, “An Abstract Semilinear Volterra Integrodifferential Equations," Proceedings of American Mathematic Society, Vol. 69, 1978, pp. 255-260. doi:10.1090/S0002-9939-1978-0467214-4

[22] R. Ye, "Existence of Solutions for Impulsive Partial Neutral Functional Differential Equation with Infinite Delay," Nonlinear Analysis, Vol. 73, No. 1, 2010, pp. 155-162. doi:10.1016/i.na.2010.03.008 